Originally published as:

Rattay, P., Lampert, T., Neuhauser, H., Ellert, U.

Bedeutung der familialen Lebenswelt für die Gesundheit von Kindern und Jugendlichen : Ergebnisse des Kinder- und Jugendgesundheitssurveys (KiGGS) (2012) Zeitschrift fur Erziehungswissenschaft, 15 (1), pp. 145-170.

DOI: $10.1007 / \mathrm{s} 11618-012-0261-4$

This is an author manuscript.

The definitive version is available at: http://link.springer.com/ 


\title{
Bedeutung der familialen Lebenswelt für die Gesundheit von Kindern und Jugendlichen
}

\section{Ergebnisse des Kinder- und Jugendgesundheitssurveys (KiGGS)}

\author{
Petra Rattay · Thomas Lampert · Hannelore Neuhauser · Ute Ellert
}

Robert Koch-Institut, Abt. Epidemiologie und Gesundheitsberichterstattung, Postfach 6502 61, 13302 Berlin, Deutschland

E-Mail: RattayP@rki.de

\section{Zusammenfassung:}

Die Familie ist im Kindes- und Jugendalter die zentrale Sozialisationsinstanz. Aber welche Faktoren der familialen Lebenswelt sind für die Gesundheit von Kindern und Jugendlichen in welcher Lebensphase von Bedeutung? Der Frage wird am Beispiel der durch die Eltern eingeschätzten allgemeinen Gesundheit nachgegangen. Datenbasis bildet die für Deutschland repräsentative KiGGS-Studie 2003-2006 ( $n=17.641$, Alter: 0 bis 17 Jahre). Zur Quantifizierung der Effekte der familialen Faktoren auf die Gesundheit werden - stratifiziert nach fünf Altersgruppen und Geschlecht - binär logistische Regressionsanalysen gerechnet. Einbezogen werden strukturelle, soziallagen- und herkunftsbezogene Faktoren von Familie sowie die Wohnsituation und das Familienklima. Die bivariate Analyse belegt, dass nahezu alle Faktoren (zumindest in einzelnen Altersgruppen) Einfluss auf die allgemeine Gesundheit haben. In der multivariaten Analyse zeigt sich, dass insbesondere dem Familienklima eine wichtige Bedeutung für eine gute Gesundheit zukommt. Bei den Jungen besitzt bis zum Beginn des Jugendalters zudem ein beidseitiger Migrationshintergrund einen ungünstigen Einfluss auf eine gute allgemeine Gesundheit.

Schlüsselwörter: Familie · Allgemeiner Gesundheitszustand · Kinder · Jugendliche · KiGGS

\section{Significance of family life for the health of children and adolescents - Results of the German Health Interview and Examination Survey for Children and Adolescents (KiGGS)}

\begin{abstract}
:
Family is the central place of socialization during childhood and adolescence. What are the determining factors of family life that are important for the health of children and adolescents and in which stages of their life do these factors take effect? The question is answered with reference to the general health of children and adolescents as rated by their parents. The analysis is conducted with data from the KiGGS study 2003-2006 which is representative of Germany ( $n=17641$, age: $0-17$ years). The gender-specific effects of different family factors on good health are analysed in five age groups using binary logistic regression analysis including structural, socioeconomic and origin-related factors of family as well as housing conditions and family cohesion. Bivariate analysis indicates significant effects on general health for almost all included factors (at least in some age groups). Multivariate analysis shows family cohesion to be the most important factor for good health. In boys a bilateral migration background also has an adverse effect on good general health until the beginning of adolescence.
\end{abstract}

Keywords: Family · General health · Children · Adolescents · KiGGS 
In den Erziehungswissenschaften finden die Themen Gesundheit - verstanden als physisches, psychisches und soziales Wohlbefinden - und Gesundheitsförderung zunehmend Berücksichtigung, da deutlich wird, dass eine gesunde Entwicklung und Sozialisations- und Lernprozesse eng miteinander verwoben sind. So beschäftigt sich u. a. der 13. Kinder- und Jugendbericht mit dem Thema „Mehr Chancen für gesundes Aufwachsen“ und der Möglichkeit der Gesundheitsförderung in der Kinder- und Jugendhilfe (Bundesministerium für Familie, Senioren, Frauen und Jugend 2009). In der Diskussion um die Gesundheitsförderung in „pädagogischen Settings“ (Bals et al. 2008) steht - neben Kindertagesstätte und Schule insbesondere die familiale Lebenswelt im Vordergrund.

Der vorliegende Beitrag beschäftigt sich mit der Frage, welche familialen Faktoren für die allgemeine Gesundheit von Kindern und Jugendlichen in unterschiedlichen Lebensphasen von Bedeutung sind. Aufbauend auf den Ergebnissen wird skizziert, welche Anknüpfungspunkte für eine familienorientierte Gesundheitsförderung bestehen.

\section{Forschungsstand}

Die Familie bildet die zentrale Sozialisationsinstanz und nimmt damit großen Einfluss auf die Gesundheit. In der Familie bekommen Kinder von klein auf bis ins Jugendalter den Umgang mit ihrem Körper, mit Gesundheit und Krankheit sowie Bewältigungsstrategien bei gesundheitlichen Belastungen vermittelt (Kolip 1998). Gerade in den alltäglichen familialen Interaktionen und über die Zugänge, die Familie zu anderen Erfahrungswelten eröffnet, erwerben die Heranwachsenden grundlegende Einstellungen, Fähigkeiten und Kenntnisse, die maßgeblich zu ihrer psychischen, körperlichen, sozialen, kognitiven und somit gesunden Entwicklung beitragen (Bundesministerium für Familie, Senioren, Frauen und Jugend 2005). Die gesundheitsbezogene Sozialisation erfolgt insbesondere über familiale Alltagspraktiken wie die Gestaltung der Mahlzeiten, das Bewegungs- und Freizeitverhalten in der Familie, feste Tageszeiten für das Aufstehen und Ins-Bett-Gehen, die Inanspruchnahme von medizinischen Leistungen sowie über familiale Haltungen zum Beispiel zur Hygiene. Aber auch gesundheitsabträglichen Praktiken wie dem Konsum von Tabak und Alkohol sind Heranwachsende in ihrer Familie mehr oder weniger direkt ausgesetzt.

Als wichtigste protektive Faktoren von Familie in Hinblick auf das gesundheitliche Wohlergehen eines Kindes werden eine dauerhaft gute Beziehung zu primären Bezugspersonen und ein sicheres Bindungsverhalten eingestuft (Egle et al. 2002). Darüber hinaus werden auch ein auf Selbstständigkeit orientiertes Erziehungsklima, ein positives Familienklima, enge Geschwisterbeziehungen, eine gute finanzielle Ausstattung und ein hoher Bildungsgrad der Eltern als gesundheitsfördernd eingeschätzt (Hurrelmann 2006). Als familiale Risikofaktoren für die Gefährdung der kindlichen Entwicklung werden ungünstige materielle Bedingungen, schlechte Schulbildung der Eltern, Arbeitslosigkeit, eine hohe Geschwisteranzahl, beengte Wohnverhältnisse, chronische Disharmonie der Eltern, autoritäres elterliches Verhalten, schwere körperliche Erkrankung oder psychische Störung eines Elternteils, eine verminderte Verfügbarkeit der Bindungsperson(en) insbesondere im Säuglings- und Kleinkindalter bzw. eine emotional schlechte Eltern-Kind-Beziehung sowie Vernachlässigung und Gewalt diskutiert. Die genannten familialen Schutz- und Risikofaktoren greifen über komplexe Prozesse ineinander, so dass weniger einzelne Faktoren als vielmehr die Summe der verschiedenen Faktoren Einfluss auf das Wohlergehen eines Kindes hat (Egle et al. 2002). 
Inwieweit variiert aber nun der familiale Einfluss auf die Gesundheit mit dem Alter der Heranwachsenden? Anknüpfend an die im Jugendalter erfolgende Ablösung von der Herkunftsfamilie und die gleichzeitig einsetzende starke Orientierung an Gleichaltrigen wird in den Jugendgesundheitswissenschaften die These der gesundheitlichen Angleichung im Jugendalter diskutiert, die insbesondere von West $(1988,1997)$ angestoßen wurde: Während in der frühen Kindheit Unterschiede in der Gesundheit in relativ hohem Maße von sozioökonomischen Faktoren beeinflusst werden, verschwinden im frühen Jugendalter die Unterschiede zwischen den sozioökonomischen Gruppen fast völlig. Insbesondere Schule, Peergroup und Jugendkultur besitzen einen nivellierenden Einfluss auf die gesundheitliche Ungleichheit im frühen Jugendalter. Mit Eintritt ins Berufsleben setzen dann soziale Schließungsprozesse ein, so dass soziale Ungleichheiten wieder an Bedeutung für die Gesundheit gewinnen.

Zahlreiche Studien präzisieren allerdings das Bild dahingehend, dass die Bedeutung des sozioökonomischen Status im Lebensverlauf in starkem Maße von dem gewählten Gesundheits-Outcome abhängt: So zeigen sich im frühen Jugendalter bei leichter verlaufenden Erkrankungen, bei nicht-tödlichen Unfällen sowie bei psychosomatischen und emotionalen Problemen nur schwache oder keine Zusammenhänge mit dem sozioökonomischen Status (Chen et al. 2002; Piko \& Fitzpatrick 2001; Rahkonen et al. 1995; Siahpush \& Singh 2000; Vuille \& Schenkel 2001; West 1988; West et al. 1990). Demgegenüber weisen tödliche Unfälle sowie schwere chronische Erkrankungen auch im Jugendalter einen deutlichen sozialen Gradienten auf (West 1997).

Für die durch die Jugendlichen selbst oder durch die Eltern eingeschätzte allgemeine Gesundheit stellt sich die Forschungslage dagegen recht inkonsistent dar: Mit Blick auf Veränderungen über die Lebensspanne vom Säugling bis zum Jugendlichen finden sich überwiegend Studien, die auf die Bedeutung sozioökonomischer Einflussfaktoren - insbesondere des Einkommens - für den durch die Eltern eingeschätzten allgemeinen Gesundheitsstatus fokussieren. Während Eltern mit niedrigem Einkommen in allen Studien von einer schlechteren Gesundheit ihrer Kinder berichten, kann die Frage nach der Variation des Einflusses sozioökonomischer Faktoren auf die allgemeine Gesundheit mit dem Alter der Heranwachsenden nicht eindeutig beantwortet werden. So kommen Chen et al. (2006), Halldorsson et al. (2000) und Burgess et al. (2004) zu dem Ergebnis, dass der Einkommensgradient unabhängig vom Alter relativ konstant ist. Case et al. (2002), Case und Paxson (2002), Murasko (2008) und Khanam et al. (2009) können hingegen belegen, dass die Bedeutung des Einkommens für die durch die Eltern als schlecht eingeschätzte Gesundheit mit dem Alter zunimmt. Des Weiteren gibt es Studien, die aufzeigen, dass der Zusammenhang zwischen Einkommen und der subjektiven allgemeinem Gesundheit über die Altersspanne nicht linear verläuft (Currie et al. 2007; Harris et al. 2008; Spencer 2006). Belege für die von West postulierte These der Nivellierung gesundheitlicher Ungleichheit im Jugendalter finden sich hingegen eher in Studien, die auf Selbstauskünften der Jugendlichen zum familialen Wohlstand fußen (Goodman 1999; Mullan \& Currie 2000; Newacheck 1989; Torsheim et al. 2004; Vingilis et al. 1998).

Für andere familiale Faktoren - wie Bildungs- und Erwerbsstatus der Eltern, Familienform, Wohnsituation oder kulturelle Herkunft der Familie - wurde hingegen deutlich seltener untersucht, ob ihre Bedeutung für die allgemeine Gesundheit mit dem Alter der Heranwachsenden variiert. Vielfach erfolgte in Studien zur Altersabhängigkeit des ökonomischen Einflusses auf die allgemeine Gesundheit lediglich eine Adjustierung für die genannten Faktoren. In der Gesamtschau der internationalen Forschungsergebnisse scheinen elterliche Bildung und Arbeitslosigkeit sowie die Familienform im Kleinkind- und Vorschulalter kaum eine 
Bedeutung für die allgemeine Gesundheit zu besitzen (Burgess et al. 2004; Coneus \& Spiess 2008; Dowd 2007; Khanam et al. 2009). Für das Grundschul- und Jugendalter kommen die Studien zu differierenden Ergebnissen hinsichtlich ihrer Bedeutung für die allgemeine Gesundheit (Call \& Nonnemaker 1999; Emerson et al. 2006; Goodman 1999; Klocke \& Becker 2003; Siahpush \& Singh 2000; Spencer 2006; van der Lucht et al. 1992).

Neben dem Einkommen kommt der Gesundheit der Eltern - insbesondere der Gesundheit der Mutter - große Bedeutung für den allgemeinen Gesundheitszustand der Heranwachsenden zu (Burgess et al. 2004; Case et al. 2002; Case \& Paxson 2002; Coneus \& Spiess 2008; Currie et al. 2007; Dowd 2007; Khanam et al. 2009; Watanabe et al. 2006). Es lassen sich für alle Altersbereiche Studien finden, die den Zusammenhang zwischen der Gesundheit der Mutter und der des Kindes untermauern. Anders als für das Einkommen werden in den Studien für die Gesundheit der Mutter allerdings meist keine altersstratifizierten Ergebnisse berichtet. Inwieweit die Bedeutung der Gesundheit der Mutter mit dem Alter der Heranwachsenden zu- oder abnimmt, ist somit bisher nicht beantwortet. Vereinzelt findet auch der Body-Mass-Index von Mutter und Vater Eingang in die Analyse des allgemeinen Gesundheitsstatus; allerdings mit divergierenden Ergebnissen (Burgess et al. 2004; Case et al. 2002). Ähnliches gilt für das elterliche Gesundheitsverhalten: Uneinheitliche Befunde zeigen sich beim Tabakkonsum der Eltern, beim elterlichen Verzehr von Obst und Gemüse, bei der körperlichen Aktivität der Eltern sowie mit Blick auf das Stillen (Burgess et al. 2004; Case et al. 2002; Case \& Paxson 2002; Currie et al. 2007; Khanam et al. 2009).

Zum Einfluss des Familienklimas bzw. der Qualität der Eltern-Kind-Beziehung auf den allgemeinen Gesundheitsstatus finden sich nur wenige Studien. Da diese ausschließlich auf Befragungen von Jugendlichen beruhen, erlauben sie nur für das Jugendalter Aussagen zum Zusammenhang zwischen Familienklima und allgemeiner Gesundheit, nicht aber für das Kindesalter. In Abhängigkeit vom gemessenen Konstrukt zeigen sich teilweise signifikante Ergebnisse: Jugendliche, die eine gute Beziehung zu ihren Eltern sowie eine hohe elterliche Unterstützung berichten, fühlen sich subjektiv gesünder als Gleichaltrige, auf die dies nicht zutrifft (Almgren et al. 2009; Call \& Nonnemaker 1999; Vingilis et al. 1998).

Der Überblick über den Forschungsstand zeigt, dass nur wenige Studien den Einfluss unterschiedlicher familialer Faktoren auf die durch die Eltern eingeschätzte allgemeine Gesundheit von Kindern und Jugendlichen nach Alter differenziert über die gesamte Spanne von 0 bis 17 Jahren untersuchen. Die These der Abschwächung des familialen Einflusses auf die Gesundheit im frühen Jugendalter wird ferner überwiegend für sozioökonomische Faktoren diskutiert, während andere familiale Einflussgrößen weitgehend unberücksichtigt bleiben. Werden über die sozioökonomische Situation hinausgehende familiale Faktoren in Studien berücksichtigt, gehen diese vielfach lediglich als Confounder-Variablen ein, ohne dass für sie unadjustierte und nach Alter stratifizierte Ergebnisse berichtet werden.

\section{Fragestellung}

Die hier vorgestellte Analyse geht der Frage nach, welche familialen Faktoren in welcher Lebensphase der Kindheit und Jugend für die durch die Eltern eingeschätzte allgemeine Gesundheit von Bedeutung sind. Neben der sozioökonomischen Situation der Familie werden strukturelle, herkunfts- und gesundheitsbezogene Faktoren sowie die Wohnsituation und das Familienklima in die Analyse einbezogen. Es wird versucht, die in der internationalen Forschung bestehende Lücke einer Quantifizierung der altersabhängigen Bedeutung unterschiedlicher familialer Faktoren für das Alter von 0 bis 17 Jahren zu schließen. 
Einerseits wird angenommen, dass familiale Faktoren über die gesamte Spanne von Kindheit und Jugend einen prägenden Einfluss auf die in ihr Heranwachsenden und deren Gesundheit haben. Andererseits wird vermutet, dass die Bedeutung einzelner Faktoren der familialen Lebenswelt für die Gesundheit nicht statisch ist, sondern über die Altersspanne der Kindheit und Jugend variiert bzw. mit der im Jugendalter erfolgenden Ablösung von den Eltern abnimmt.

Da sich in der Morbidität im Kindes- und Jugendalter teils deutliche Unterschiede zwischen Mädchen und Jungen zeigen, wird zudem analysiert, ob sich hinsichtlich des Stellenwerts einzelner familialer Faktoren für die Gesundheit Unterschiede nach Geschlecht zeigen.

Die Fragestellung wird im vorliegenden Beitrag für den durch die Eltern eingeschätzten allgemeinen Gesundheitszustandes untersucht. Die selbst eingeschätzte Gesundheit erweist sich mit Blick auf Erwachsene international als guter Prädiktor für Morbidität, Mortalität und die Inanspruchnahme von Leistungen des Gesundheitswesens (Idler \& Benyamini 1997) und spiegelt zudem gesundheitsbezogene Einstellungen, Wahrnehmungen und Bewertungen wider, die häufig auf sozialen Vergleichen und der Einschätzung der individuellen Entscheidungs- und Handlungsmöglichkeiten basieren (Lampert et al. 2010). Insbesondere im frühen Kindesalter werden Gesundheit und Krankheit aber fast ausschließlich durch die Eltern definiert und sind im starken Maße durch deren Einstellungen und Wahrnehmungen geprägt.

\section{Methoden}

\subsection{Datenbasis}

Der vorliegende Beitrag basiert auf Daten des Kinder- und Jugendgesundheitssurveys (KiGGS), der im Zeitraum zwischen Mai 2003 und Mai 2006 durch das Robert Koch-Institut durchgeführt wurde (Kurth 2007). Er stellt erstmals für Deutschland bundesweit repräsentative Daten zum Gesundheitszustand von Kindern und Jugendlichen im Alter von 0 bis 17 Jahren bereit und wird derzeit als Kohortenstudie fortgeführt.

Die Stichprobenziehung erfolgte nach einem zweistufig geschichteten Auswahlverfahren: Im ersten Schritt wurden 167 Untersuchungsorte ermittelt, die für die sozialräumliche Struktur in Deutschland repräsentativ sind. Im zweiten Schritt wurden dann für die ausgewählten Städte und Gemeinden über die Einwohnermelderegister per Zufallsverfahren die Teilnehmer(innen) bestimmt (Kamtsiuris et al. 2007). An der Studie nahmen insgesamt 17.641 Kinder und Jugendliche teil (Teilnahmequote: 66,6\%).

Untersuchung und Befragung der ausgewählten Kinder und Jugendlichen fanden in Begleitung ihrer Eltern in vor Ort eingerichteten Studienzentren statt. Dort wurden zum einen die Eltern aller Altersgruppen sowie die Kinder und Jugendlichen ab dem Alter von 11 Jahren mittels Selbstausfüllfragebögen befragt. Themen der schriftlichen Befragungen waren die körperliche und psychische Gesundheit, das Gesundheitsverhalten des Kindes, die Inanspruchnahme von Leistungen der Gesundheitsversorgung sowie einzelne Aspekte der sozialen Lebensbedingungen. Zum anderen wurden die Kinder medizinisch untersucht sowie mit den Eltern ein ärztliches Interview zu Krankheiten, Impfungen und Arzneimittelgebrauch ihres Kindes durchgeführt.

\subsection{Erhebungsinstrumente}

Outcome. Die Einschätzung des allgemeinen Gesundheitszustandes liegt in KiGGS für die Altersgruppe der 0- bis 17-Jährigen zum einen als Einschätzung durch die Eltern und zum 
anderen für die Altersgruppe der 14- bis 17-Jährigen als Selbsteinschätzung der Jugendlichen vor. Für die vorliegende Analyse wurde lediglich auf die Elternauskünfte zurückgegriffen, um über den gesamten Altersverlauf hinweg Daten der gleichen Messsituation analysieren zu können. Die durch die Eltern eingeschätzte allgemeine Gesundheit wurde in der schriftlichen Befragung mit folgender Frage erhoben: „Wie würden Sie den Gesundheitszustand Ihres Kindes im Allgemeinen beschreiben?" Als Antwortmöglichkeit stand eine 5-stufige Skala mit den Vorgaben „sehr gut" bis „sehr schlecht" zur Verfügung. Für die vorgestellte Analyse wurden die Antworten „sehr gut“ und „gut" sowie „mittelmäßig“ bis „sehr schlecht" zusammengefasst, so dass die Variable die dichotome Ausprägung „gut" und „nicht gut“ besitzt. Insgesamt liegen für 8.486 Mädchen und 8.906 Jungen Einschätzungen der Gesundheit durch ein Elternteil vor.

Prädiktoren. KiGGS liefert zur Abbildung der familialen Lebenswelt zahlreiche Indikatoren, die aber keineswegs alle in der Literatur diskutierten Risiko- und Schutzfaktoren abbilden. Der vorliegende Beitrag fokussiert insbesondere auf Indikatoren der elterlichen Lebenslage. Um in die multivariate Betrachtung der Prädiktoren auch Eineltern- und Stieffamilien einbeziehen zu können, wurde auf haushaltsbezogene Konstrukte zurückgegriffen.

Ausgehend von den Antworten der Eltern zum Hauptaufenthaltsort ihres Kindes wurde die Variable „Zusammenwohnen mit beiden leiblichen Elternteilen (ja/nein)“ gebildet. Darüber hinaus wird die Anzahl der Geschwister einbezogen, die sich auf alle im Haushalt lebenden Geschwister bezieht (auch Halb- oder Stiefgeschwister).

Zur Abbildung der familialen Wohnsituation stehen in KiGGS Angaben der Eltern zur Größe der Wohnung zur Verfügung. Diese wurde in Beziehung zur Anzahl der Personen im Haushalt gesetzt.

Die Erhebung des Migrationsstatus erlaubt die Differenzierung zwischen beid- und einseitigem sowie keinem Migrationshintergrund. Ein beidseitiger Migrationshintergrund liegt vor, wenn Kinder und Jugendliche selbst aus einem anderen Land zugewandert sind und mindestens ein Elternteil nicht in Deutschland geboren ist, oder beide Eltern zugewandert und/oder nichtdeutscher Staatsangehörigkeit sind. Kinder und Jugendliche mit einseitigem Migrationshintergrund sind in Deutschland geboren und haben ein Elternteil, das aus einem anderen Land zugewandert und/oder nichtdeutscher Staatangehörigkeit ist (Schenk et al. 2008).

Die sozioökonomische Lage der Familie wird durch die Indikatoren Erwerbs- und Sozialstatus der Eltern abgebildet. Die Variablen wurden für den Gesamthaushalt unter Einbezug der im Haushalt lebenden Elternteile gebildet. Beim Erwerbsstatus wird differenziert, ob mindestens ein Elternteil Vollzeit beschäftigt ist oder nicht. Der Sozialstatus wird über einen mehrdimensionalen aggregierten Index erfasst, der auf der Basis der Angaben der Eltern zu ihrer schulischen und beruflichen Qualifikation, zu ihrer beruflichen Stellung und zum Haushaltsnettoeinkommen berechnet wurde. Für die Analysen wird zwischen „niedriger" , "mittlerer" und "hoher" Statusgruppe unterschieden (Lange et al. 2007).

Ein Indikator, der in KiGGS zur Abbildung der Gesundheit der Eltern zur Verfügung steht, ist der Body-Mass-Index von Mutter und Vater, der auf Selbstangaben der Eltern zu Körpergröße und -gewicht beruht. Aus diesen Angaben wurde die dichotome Variable „Übergewicht mindestens eines Elternteils (ja/nein)" konstruiert.

Mit Blick auf das Gesundheitsverhalten der Eltern liegen in KiGGS u. a. Elternangaben zum Tabakkonsum in der Wohnung, zur Stilldauer und zur Inanspruchnahme von U-Untersuchungen vor. Die Angaben zum Tabakkonsum in der Wohnung wurden auf die Ausprägungen „ja“/,nein“ dichotomisiert. Zur Stilldauer wurde aus den vorliegenden Daten eine Variable mit den Ausprägungen „Kind wurde 6 Monate oder länger voll gestillt“, „Kind wurde gestillt, 
aber nicht 6 Monate voll“ und „Kind wurde nie gestillt" gebildet. Die KiGGS-Daten zu den UUntersuchungen wurden für Kinder und Jugendliche ab dem 2. Lebensjahr zur Variablen „vollständige Inanspruchnahme der U1- bis U7-Untersuchungen (ja/nein)“ verdichtet.

Das Familienklima wurde mit einer modifizierten Skala nach Schneewind et al. (1985) erfragt und beinhaltet Angaben zum familialen Zusammenhalt, zur gemeinsamen Freizeitgestaltung sowie zur familialen Kontrolle. Eine elterliche Einschätzung des Familienklimas liegt für die 3- bis 17-Jährigen vor. Für die Bildung des Indexes wurden die Itemwerte summiert und in Werte zwischen 0 und 10 transformiert. In der multivariaten Analyse stehen niedrige Werte für ein gutes Familienklima. Für die bivariate Analyse erfolgte zudem eine Kategorisierung der Skalenwerte entlang der 80. und 90. Perzentile in "keine“, „geringe " und „große“ Defizite (Erhart et al. 2007).

\subsection{Statistische Analysen}

Im ersten Schritt wurden entlang der familialen Einflussgrößen Prävalenzen des durch die Eltern eingeschätzten Gesundheitszustandes der Kinder und Jugendlichen berechnet. Des Weiteren wurden in der bivariaten Analyse mittels binär logistischer Regressionen für die einzelnen Prädiktoren Odds Ratios und deren 95\%-Konfidenzintervalle geschätzt, um die Bedeutung der einzelnen Faktoren der familialen Lebenswelt für die subjektive Gesundheit der Kinder und Jugendlichen quantifizieren zu können. Durch den Bericht der unadjustierten Werte ist eine direkte Vergleichbarkeit der Ergebnisse mit anderen Studien gegeben.

Abschließend wurden in der multivariaten Analyse die auf der Basis der bivariaten Ergebnisse als relevant erachteten Prädiktoren gleichzeitig in der logistischen Modellierung der nicht guten Gesundheit (ja/nein) berücksichtigt. Bei den Prädiktoren diente jeweils die in der bivariaten Betrachtung mit einem guten Gesundheitszustand assoziierte Kategorie als Referenzgruppe. Odds Ratios, die über dem Wert 1 liegen, beziffern somit, um welchen Faktor die "Chance“, bei nicht guter Gesundheit zu sein, im Verhältnis zur Referenzgruppe höher ist. Für die logistischen Regressionen werden neben den Odds Ratios die 95\%-Konfidenzintervalle sowie die Werte für Nagelkerkes $\mathrm{R}^{2}$ ausgewiesen.

Alle Analysen wurden nach Altersgruppen (0-2 Jahre, 3-6 Jahre, 7-10 Jahre, 11-13 Jahre und 14-17 Jahre) und Geschlecht stratifiziert durchgeführt. Da für die 0- bis 2-Jährigen keine Angaben zum Familienklima sowie zur Vollständigkeit der Inanspruchnahme der U-Untersuchungen vorliegen, konnten diese Variablen nicht in die multivariate Modellierung für diese Altersgruppe eingehen. Die für dieses Alter berichteten Odds Ratios der multivariaten Modellierung sind daher nicht direkt mit denen der anderen Altersgruppen vergleichbar.

Die statistischen Analysen wurden mit einem Gewichtungsfaktor durchgeführt, der Abweichungen der Netto-Stichprobe von der Bevölkerungsstruktur (Stand: 31.12.2004) hinsichtlich Alter, Geschlecht, Region (Ost/West/Berlin) und Staatsangehörigkeit korrigiert und so repräsentative Aussagen ermöglicht. Um die Korrelation der Proband(inn)en innerhalb einer Gemeinde zu berücksichtigen, wurden die Analysen mit den SPSS-17-Verfahren für komplexe Stichproben berechnet. 
Tab. 1: Beschreibung der Stichprobe

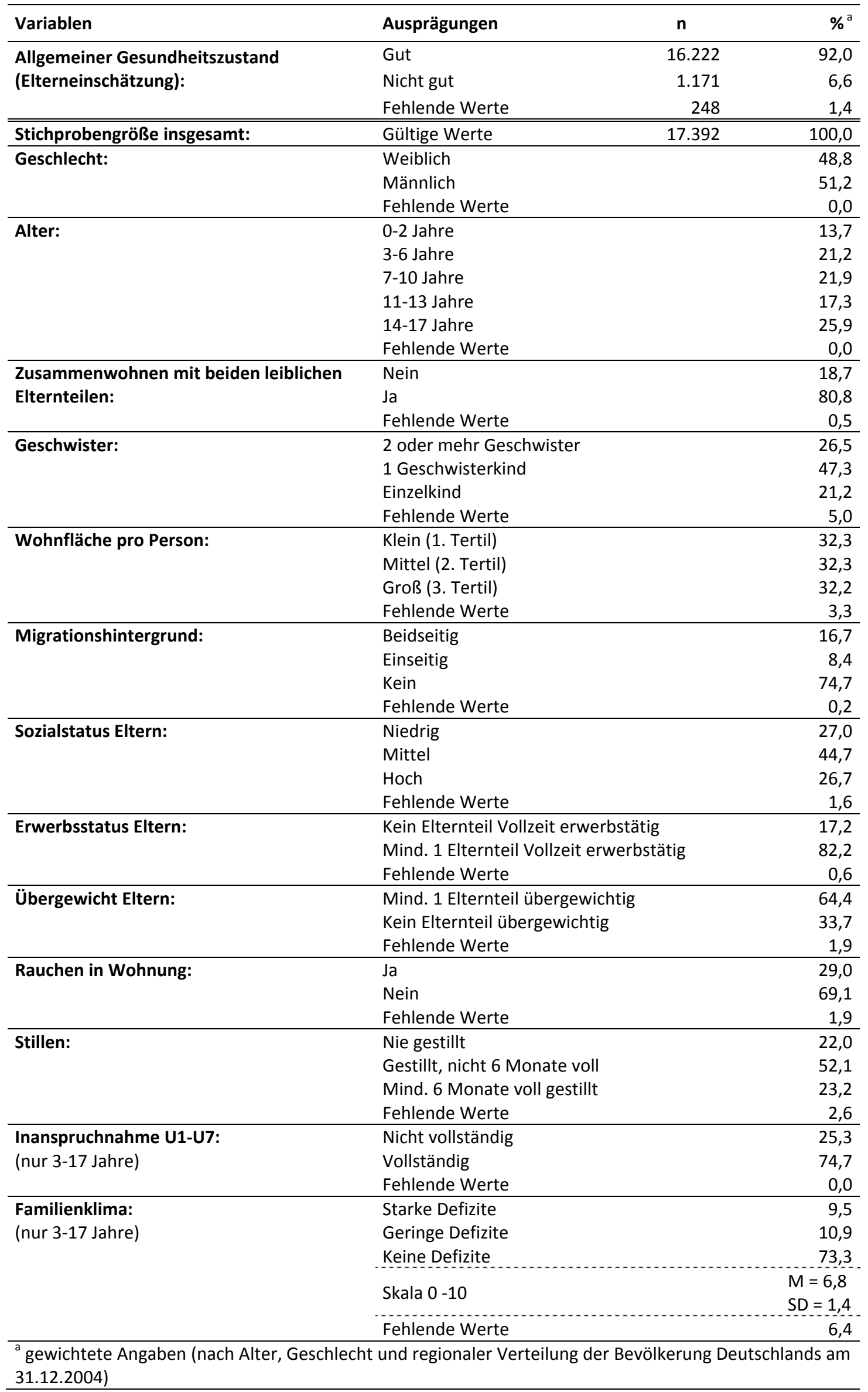




\section{Ergebnisse}

In Tab. 2 sind die prozentualen Häufigkeiten der durch die Eltern als nicht gut eingeschätzten Gesundheit von Kindern und Jugendlichen im Altersverlauf nach Geschlecht stratifiziert dargestellt. Bei beiden Geschlechtern zeigt sich ein signifikanter Alterseffekt. Im Säuglings- und frühen Kleinkindalter schätzen nur 2,9\% der Eltern die Gesundheit ihres Kindes als nicht gut ein. Im Alter von 3 bis 6 Jahren steigt der Anteil der Kinder mit einer nicht guten Gesundheit auf 6,7 \% an. Während bei den Mädchen der Anteil der Nicht-Gesunden bis zum Alter von 13 Jahren auf nahezu konstantem Niveau bleibt und erst in der Altersgruppe der 14- bis 17Jährigen auf 9,8\% ansteigt, zeigt sich bei den Jungen kein geradliniger Verlauf. Auffällig ist, dass der Anteil der nicht gesunden Jungen im Grundschulalter tendenziell geringer ist als im Vorschulalter und im Alter von 11 bis 13 Jahren in statistisch signifikantem Maße ansteigt, um dann bis zum Ende des Jugendalters auf diesem Niveau zu verbleiben.

Tab. 2: Nicht guter allgemeiner Gesundheitszustand bei Kindern und Jugendlichen im Elternurteil stratifiziert nach Alter und Geschlecht (Prävalenzen in \% und 95\%-Konfidenzintervalle)

\begin{tabular}{lcccccccccc}
\hline & \multicolumn{2}{c}{$\mathbf{0 - 2}$ Jahre } & \multicolumn{2}{c}{ 3-6 Jahre } & \multicolumn{2}{c}{ 7-10 Jahre } & \multicolumn{2}{c}{ 11-13 Jahre } & \multicolumn{2}{c}{ 14-17 Jahre } \\
& $\%$ & $\mathbf{( 9 5 \% - K I )}$ & \% & $\mathbf{( 9 5 \% - K I )}$ & \% & $\mathbf{( 9 5 \% - K I )}$ & \% & $\mathbf{( 9 5 \% - K I )}$ & \% & (95\%-KI) \\
\hline Jungen & 3,6 & $(2,6-4,9)$ & 7,7 & $(6,3-9,3)$ & 5,6 & $(4,6-6,8)$ & 9,0 & $(7,2-11,1)$ & 8,7 & $(7,4-10,2)$ \\
Mädchen & 2,1 & $(1,5-3,1)$ & 5,6 & $(4,5-6,9)$ & 5,7 & $(4,6-7,0)$ & 5,8 & $(4,6-7,2)$ & 9,8 & $(8,4-11,5)$ \\
Insgesamt & 2,9 & $(2,3-3,6)$ & 6,7 & $(5,7-7,8)$ & 5,7 & $(4,9-6,5)$ & 7,4 & $(6,3-8,7)$ & 9,3 & $(8,3-10,4)$ \\
\hline
\end{tabular}

In den Tab. 3 und 4 sind die Ergebnisse der bivariaten Analysen stratifiziert nach Alter und Geschlecht dargestellt.

Das Zusammenleben mit beiden leiblichen Elternteilen ist bei Jungen nur im Alter von 3 bis 6 Jahren und bei Mädchen im Alter von 11 bis 17 Jahren in statistisch signifikantem Maße mit einer guten allgemeinen Gesundheit assoziiert.

Bei den Jungen schneiden im Säuglings- und frühen Kleinkindalter Einzelkinder gesundheitlich signifikant besser ab als Kinder mit Geschwistern; in allen anderen Altersgruppen und bei den Mädchen zeigen sich keine signifikanten Unterschiede.

Jungen, die in eher beengten Wohnverhältnissen aufwachsen, weisen über die Altersspanne von 0 bis 13 Jahren eine signifikant schlechtere Gesundheit auf als Jungen, die in Wohnungen mit einer großen Wohnfläche pro Haushaltsmitglied leben. Lediglich im Alter von 14 bis 17 Jahren kommt der Wohnungsgröße keine Bedeutung zu. Bei den Mädchen zeigen sich erst ab dem Grundschulalter Unterschiede zu Ungunsten derjenigen, die in eher kleinen Wohnungen leben.

Jungen mit beidseitigem Migrationshintergrund besitzen in allen Altersgruppen seltener eine gute Gesundheit als Jungen ohne Migrationshintergrund. Bei den Mädchen ist ein ähnlicher Trend zu verzeichnen; signifikante Unterschiede zeigen sich jedoch nur im Vorschul-, Grundschul- und Jugendalter. Signifikante Unterschiede zwischen Kindern mit einseitigem und keinem Migrationshintergrund lassen sich nur bei den 3- bis 6-jährigen Mädchen feststellen. 
Tab. 3: Bivariate Analyse der Bedeutung familialer Faktoren für eine nicht gute Gesundheit von Jungen, stratifiziert nach Altersgruppen (Prävalenzen in \%, Odds Ratios und 95\%-Konfidenzintervalle)

\begin{tabular}{|c|c|c|c|c|c|c|c|c|c|c|}
\hline \multirow{2}{*}{ Jungen } & \multicolumn{2}{|r|}{ 0-2 Jahre } & \multicolumn{2}{|r|}{ 3-6 Jahre } & \multicolumn{2}{|r|}{ 7-10 Jahre } & \multicolumn{2}{|r|}{ 11-13 Jahre } & \multicolumn{2}{|r|}{ 14-17 Jahre } \\
\hline & $\%$ & OR (95\%-KI) & $\%$ & OR (95\%-KI) & $\%$ & OR (95\%-KI) & $\%$ & OR (95\%-KI) & $\%$ & OR (95\%-KI) \\
\hline \multicolumn{11}{|l|}{$\begin{array}{l}\text { Zusammenwohnen } \\
\text { mit beiden Eltern: }\end{array}$} \\
\hline Nein & 1,8 & $0,47(0,14-1,57)$ & 11,9 & $1,82(1,07-3,08)$ & 6,7 & $1,25(0,66-2,37)$ & 10,3 & $1,22(0,80-1,87)$ & 10,1 & $1,24(0,82-1,88)$ \\
\hline Ja (Ref.) & 3,7 & 1,00 & 6,9 & 1,00 & 3,8 & 1,00 & 7,2 & 1,00 & 7,5 & 1,00 \\
\hline \multicolumn{11}{|l|}{ Anzahl Geschwister: } \\
\hline $\begin{array}{l}2 \text { oder mehr } \\
\text { Geschwister }\end{array}$ & 5,8 & $3,79(1,45-9,90)$ & 7,7 & $0,95(0,54-1,67)$ & 6,1 & $1,17(0,60-2,28)$ & 10,3 & $1,09(0,59-2,03)$ & 8,3 & $0,92(0,52-1,60)$ \\
\hline 1 Geschwisterkind & 4,6 & $2,98(1,41-6,33)$ & 7,0 & $0,86(0,52-1,42)$ & 5,2 & $0,98(0,54-1,80)$ & 7,5 & $0,77(0,43-1,39)$ & 9,0 & $1,00(0,64-1,57)$ \\
\hline Einzelkind (Ref.) & 1,6 & 1,00 & 8,0 & 1,00 & 5,3 & 1,00 & 9,5 & 1,00 & 9,0 & 1,00 \\
\hline \multicolumn{11}{|l|}{$\begin{array}{l}\text { Wohnfläche pro } \\
\text { Person: }\end{array}$} \\
\hline Klein (1. Tertil) & 5,4 & $2,66(1,12-6,30)$ & 10,4 & $1,93(1,19-3,14)$ & 8,9 & $2,79(1,57-4,96)$ & 12,9 & $2,18(1,21-3,90)$ & 11,0 & $1,35(0,90-2,00)$ \\
\hline Mittel (2. Tertil) & 2,8 & $1,33(0,48-3,68)$ & 6,3 & $1,12(0,66-1,88)$ & 4,2 & $1,25(0,68-2,30)$ & 6,9 & $1,08(0,64-1,82)$ & 6,8 & $0,79(0,49-1,27)$ \\
\hline Groß (3. Tertil) (Ref.) & 2,1 & 1,00 & 5,7 & 1,00 & 3,4 & 1,00 & 6,4 & 1,00 & 8,4 & 1,00 \\
\hline \multicolumn{11}{|l|}{$\begin{array}{l}\text { Migrationshinter- } \\
\text { grund: }\end{array}$} \\
\hline Beidseitig & 7,1 & $2,39(1,15-4,99)$ & 15,1 & $2,70(1,80-4,05)$ & 11,4 & $2,81(1,78-4,43)$ & 19,4 & $3,62(2,34-5,60)$ & 12,9 & $1,72(1,07-2,77)$ \\
\hline Einseitig & 1,7 & $0,55(0,18-1,68)$ & 5,1 & $0,82(0,35-1,92)$ & 5,0 & $1,15(0,49-2,71)$ & 11,2 & $1,89(0,96-3,73)$ & 8,1 & $1,02(0,52-2,00)$ \\
\hline Kein (Ref.) & 3,1 & 1,00 & 6,2 & 1,00 & 4,4 & 1,00 & 6,2 & 1,00 & 7,9 & 1,00 \\
\hline \multicolumn{11}{|l|}{ Sozialstatus Eltern: } \\
\hline Niedrig & 5,0 & $1,48(0,65-3,37)$ & 10,9 & $3,48(1,87-6,48)$ & 7,5 & $2,08(1,13-3,83)$ & 12,8 & $2,60(1,41-4,78)$ & 10,5 & $1,56(0,94-2,61)$ \\
\hline Mittel & 2,7 & $0,76(0,33-1,76)$ & 8,1 & $2,53(1,30-4,91)$ & 5,5 & $1,47(0,86-2,51)$ & 7,8 & $1,49(0,83-2,70)$ & 8,5 & $1,23(0,76-2,01)$ \\
\hline Hoch (Ref.) & 3,5 & 1,00 & 3,4 & 1,00 & 3,8 & 1,00 & 5,3 & 1,00 & 7,0 & 1,00 \\
\hline \multicolumn{11}{|l|}{ Erwerbsstatus } \\
\hline $\begin{array}{l}\text { Kein Elternteil } \\
\text { Vollzeit }\end{array}$ & 3,4 & $0,93(0,46-1,89)$ & 12,2 & $1,95(1,28-2,99)$ & 10,7 & $2,52(1,53-4,16)$ & 15,6 & $2,28(1,48-3,51)$ & 10,8 & $1,34(0,89-2,02)$ \\
\hline $\begin{array}{l}\text { Mind. } 1 \text { Elternteil } \\
\text { Vollzeit (Ref.) }\end{array}$ & 3,6 & 1,00 & 6,7 & 1,00 & 4,5 & 1,00 & 7,5 & 1,00 & 8,3 & 1,00 \\
\hline \multicolumn{11}{|l|}{ Übergewicht Eltern: } \\
\hline $\mathrm{Ja}$ & 3,6 & $1,09(0,59-2,00)$ & 7,8 & $1,05(0,68-1,61)$ & 5,8 & $1,07(0,69-1,66)$ & 10,1 & $1,58(0,99-2,53)$ & 9,1 & $1,21(0,78-1,87)$ \\
\hline Nein (Ref.) & 3,3 & 1,00 & 7,4 & 1,00 & 5,4 & 1,00 & 6,6 & 1,00 & 7,6 & 1,00 \\
\hline \multicolumn{11}{|l|}{$\begin{array}{l}\text { Rauchen in } \\
\text { Wohnung: }\end{array}$} \\
\hline $\mathrm{Ja}$ & 6,8 & $2,46(1,20-5,05)$ & 11,4 & $1,87(1,22-2,87)$ & 6,9 & $1,43(0,92-2,22)$ & 9,1 & $1,11(0,72-1,70)$ & 9,7 & $1,19(0,83-1,72)$ \\
\hline Nein (Ref.) & 2,9 & 1,00 & 6,4 & 1,00 & 4,9 & 1,00 & 8,3 & 1,00 & 8,2 & 1,00 \\
\hline \multicolumn{11}{|l|}{ Stillen: } \\
\hline Nie gestillt & 3,4 & $0,83(0,33-2,07)$ & 10,7 & $2,08(1,18-3,66)$ & 4,5 & $1,25(0,62-2,51)$ & 9,4 & $1,55(0,85-2,84)$ & 9,8 & $0,93(0,50-1,74)$ \\
\hline $\begin{array}{l}\text { Gestillt, nicht } 6 \\
\text { Monate voll }\end{array}$ & 3,1 & $0,77(0,36-1,64)$ & 7,4 & $1,38(0,87-2,20)$ & 7,2 & $2,05(1,04-4,02)$ & 9,1 & $1,51(0,79-2,87)$ & 7,2 & $0,67(0,41-1,08)$ \\
\hline $\begin{array}{l}\text { Mind. } 6 \text { Mon. voll } \\
\text { gestillt (Ref.) }\end{array}$ & 4,0 & 1,00 & 5,5 & 1,00 & 3,6 & 1,00 & 6,2 & 1,00 & 10,5 & 1,00 \\
\hline \multicolumn{11}{|l|}{$\begin{array}{l}\text { Inanspruchnahme } \\
\text { U1 -U7: }\end{array}$} \\
\hline Nicht vollständig & & & 7,9 & $1,05(0,67-1,67)$ & 9,7 & $2,17(1,39-3,37)$ & 17,2 & $2,73(1,68-4,44)$ & 9,2 & $1,09(0,71-1,67)$ \\
\hline Vollständig (Ref.) & & & 7,5 & 1,00 & 4,7 & 1,00 & 7,1 & 1,00 & 8,6 & 1,00 \\
\hline \multicolumn{11}{|l|}{ Familienklima: } \\
\hline Starke Defizite & & & 16,5 & $3,04(1,33-6,98)$ & 6,8 & $1,50(0,68-3,34)$ & 17,9 & $3,31(1,91-5,74)$ & 13,9 & $2,25(1,47-3,45)$ \\
\hline Geringe Defizite & & & 10,4 & $1,80(0,85-3,81)$ & 7,1 & $1,58(0,75-3,33)$ & 8,3 & $1,37(0,71-2,63)$ & 7,2 & $1,07(0,65-1,78)$ \\
\hline Keine Defizite (Ref.) & & & 6,1 & 1,00 & 4,6 & 1,00 & 6,2 & 1,00 & 6,7 & 1,00 \\
\hline Skala $0-10$ & & & & $1,38(1,16-1,64)$ & & $1,29(1,12-1,49)$ & & $1,46(1,26-1,69)$ & & $1,26(1,10-1,44)$ \\
\hline
\end{tabular}

Signifikante Unterschiede sind durch Fettdruck hervorgehoben. 
Tab. 4: Bivariate Analyse der Bedeutung familialer Faktoren für eine nicht gute Gesundheit von Mädchen, stratifiziert nach Altersgruppen (Prävalenzen in \%, Odds Ratios und 95\%-Konfidenzintervalle)

\begin{tabular}{|c|c|c|c|c|c|c|c|c|c|c|}
\hline \multirow{2}{*}{ Mädchen } & \multicolumn{2}{|r|}{ 0-2 Jahre } & \multicolumn{2}{|r|}{ 3-6 Jahre } & \multicolumn{2}{|r|}{ 7-10 Jahre } & \multicolumn{2}{|r|}{ 11-13 Jahre } & \multicolumn{2}{|r|}{ 14-17 Jahre } \\
\hline & $\%$ & OR (95\%-KI) & $\%$ & OR (95\%-KI) & $\%$ & OR (95\%-KI) & $\%$ & OR (95\%-KI) & $\%$ & OR (95\%-KI) \\
\hline \multicolumn{11}{|l|}{$\begin{array}{l}\text { Zusammenwohnen } \\
\text { mit beiden Eltern: }\end{array}$} \\
\hline Nein & 2,1 & $0,95(0,26-3,44)$ & 6,3 & $1,15(0,59-2,23)$ & 6,7 & $1,27(0,81-2,01)$ & 9,7 & $2,19(1,27-3,77)$ & 13,0 & $1,58(1,08-2,30)$ \\
\hline Ja (Ref.) & 2,2 & 1,00 & 5,5 & 1,00 & 5,4 & 1,00 & 4,7 & 1,00 & 8,7 & 1,00 \\
\hline \multicolumn{11}{|l|}{ Anzahl Geschwister: } \\
\hline $\begin{array}{l}2 \text { oder mehr } \\
\text { Geschwister }\end{array}$ & 1,3 & $0,67(0,20-2,22)$ & 5,7 & $0,73(0,37-1,42)$ & 5,4 & $1,15(0,58-2,27)$ & 5,0 & $0,94(0,44-1,97)$ & 9,5 & $1,09(0,67-1,77)$ \\
\hline 1 Geschwisterkind & 2,3 & $1,14(0,49-2,65)$ & 4,4 & $0,55(0,29-1,03)$ & 5,4 & $1,15(0,58-2,29)$ & 6,3 & $1,19(0,61-2,31)$ & 9,6 & $1,10(0,66-1,82)$ \\
\hline Einzelkind (Ref.) & 2,0 & 1,00 & 7,7 & 1,00 & 4,8 & 1,00 & 5,3 & 1,00 & 8,8 & 1,00 \\
\hline \multicolumn{11}{|l|}{$\begin{array}{l}\text { Wohnfläche pro } \\
\text { Person: }\end{array}$} \\
\hline Klein (1. Tertil) & 1,8 & $0,88(0,33-2,36)$ & 7,7 & $1,58(0,93-2,68)$ & 10,1 & $3,20(1,84-5,56)$ & 9,3 & $2,39(1,28-4,46)$ & 12,8 & $2,07(1,35-3,19)$ \\
\hline Mittel (2. Tertil) & 2,2 & $1,07(0,39-2,94)$ & 3,8 & $0,75(0,43-1,31)$ & 3,0 & $0,88(0,43-1,79)$ & 4,6 & $1,13(0,64-2,00)$ & 10,3 & $1,63(1,04-2,55)$ \\
\hline Groß (3. Tertil) (Ref.) & 2,1 & 1,00 & 5,0 & 1,00 & 3,4 & 1,00 & 4,1 & 1,00 & 6,6 & 1,00 \\
\hline \multicolumn{11}{|l|}{$\begin{array}{l}\text { Migrationshinter- } \\
\text { grund: }\end{array}$} \\
\hline Beidseitig & 3,2 & $1,86(0,77-4,50)$ & 9,5 & $2,42(1,51-3,87)$ & 13,2 & $3,83(2,32-6,33)$ & 8,5 & $1,71(0,95-3,10)$ & 18,5 & $2,47(1,63-3,74)$ \\
\hline Einseitig & 3,3 & $1,95(0,67-5,71)$ & 9,7 & $2,49(1,45-4,26)$ & 7,4 & $2,01(0,89-4,52)$ & 4,8 & $0,93(0,33-2,65)$ & 5,9 & $0,69(0,28-1,67)$ \\
\hline Kein (Ref.) & 1,7 & 1,00 & 4,2 & 1,00 & 3,8 & 1,00 & 5,2 & 1,00 & 8,4 & 1,00 \\
\hline \multicolumn{11}{|l|}{ Sozialstatus Eltern: } \\
\hline Niedrig & 3,3 & $1,39(0,54-3,62)$ & 8,5 & $1,95(1,06-3,59)$ & 9,8 & $3,93(1,90-8,13)$ & 7,7 & $2,28(1,12-4,66)$ & 12,8 & $2,40(1,33-4,32)$ \\
\hline Mittel & 1,3 & $0,53(0,19-1,49)$ & 4,5 & $0,99(0,56-1,77)$ & 4,8 & $1,84(0,85-3,98)$ & 5,8 & $1,70(0,90-3,18)$ & 10,1 & $1,85(1,07-3,19)$ \\
\hline Hoch (Ref.) & 2,4 & 1,00 & 4,5 & 1,00 & 2,7 & 1,00 & 3,5 & 1,00 & 5,7 & 1,00 \\
\hline \multicolumn{11}{|l|}{ Erwerbsstatus } \\
\hline $\begin{array}{l}\text { Kein Elternteil } \\
\text { Vollzeit }\end{array}$ & 2,3 & $1,08(0,40-2,90)$ & 7,8 & $1,55(0,91-2,65)$ & 8,9 & $1,88(1,20-2,93)$ & 8,2 & $1,63(0,93-2,87)$ & 17,0 & $2,25(1,56-3,26)$ \\
\hline $\begin{array}{l}\text { Mind. } 1 \text { Elternteil } \\
\text { Vollzeit (Ref.) }\end{array}$ & 2,1 & 1,00 & 5,0 & 1,00 & 6,2 & 1,00 & 5,2 & 1,00 & 8,3 & 1,00 \\
\hline \multicolumn{11}{|l|}{ Übergewicht Eltern: } \\
\hline $\mathrm{Ja}$ & 2,0 & $0,76(0,33-1,74)$ & 5,1 & $0,76(0,49-1,19)$ & 5,8 & $1,16(0,72-1,86)$ & 6,5 & $1,51(0,83-2,76)$ & 9,1 & $0,85(0,59-1,22)$ \\
\hline Nein (Ref.) & 2,6 & 1,00 & 6,6 & 1,00 & 5,1 & 1,00 & 4,4 & 1,00 & 10,6 & 1,00 \\
\hline \multicolumn{11}{|l|}{$\begin{array}{l}\text { Rauchen in } \\
\text { Wohnung: }\end{array}$} \\
\hline $\mathrm{Ja}$ & 2,4 & $1,16(0,43-3,11)$ & 6,9 & $1,34(0,83-2,16)$ & 6,8 & $1,37(0,84-2,21)$ & 7,1 & $1,47(0,91-2,37)$ & 9,6 & $0,97(0,66-1,44)$ \\
\hline Nein (Ref.) & 2,1 & 1,00 & 5,2 & 1,00 & 5,1 & 1,00 & 5,0 & 1,00 & 9,8 & 1,00 \\
\hline \multicolumn{11}{|l|}{ Stillen: } \\
\hline Nie gestillt & 2,5 & $1,17(0,42-3,30)$ & 6,1 & $1,01(0,48-2,13)$ & 7,5 & $2,18(1,07-4,43)$ & 6,1 & $1,15(0,56-2,35)$ & 10,1 & $0,96(0,52-1,78)$ \\
\hline $\begin{array}{l}\text { Gestillt, nicht } 6 \\
\text { Monate voll }\end{array}$ & 2,1 & $0,99(0,39-2,52)$ & 5,2 & $0,86(0,51-1,45)$ & 5,8 & $1,67(0,90-3,07)$ & 5,9 & $1,10(0,59-2,03)$ & 9,6 & $0,91(0,54-1,54)$ \\
\hline $\begin{array}{l}\text { Mind. } 6 \text { Mon. voll } \\
\text { gestillt (Ref.) }\end{array}$ & 2,1 & 1,00 & 6,0 & 1,00 & 3,6 & 1,00 & 5,4 & 1,00 & 10,5 & 1,00 \\
\hline \multicolumn{11}{|l|}{$\begin{array}{l}\text { Inanspruchnahme } \\
\text { U1 -U7: }\end{array}$} \\
\hline Nicht vollständig & & & 5,9 & $1,11(0,70-1,75)$ & 11,4 & $2,73(1,74-4,30)$ & 8,6 & $1,76(1,05-2,98)$ & 12,6 & $1,46(1,02-2,09)$ \\
\hline Vollständig (Ref.) & & & 5,4 & 1,00 & 4,5 & 1,00 & 5,1 & 1,00 & 9,0 & 1,00 \\
\hline \multicolumn{11}{|l|}{ Familienklima: } \\
\hline Starke Defizite & & & 13,5 & $2,93(1,45-5,91)$ & 16,5 & $5,23(2,78-9,83)$ & 9,6 & $1,98(1,07-3,69)$ & 18,5 & $3,69(2,37-5,76)$ \\
\hline Geringe Defizite & & & 3,0 & $0,59(0,24-1,42)$ & 4,6 & $1,28(0,54-3,06)$ & 3,0 & $0,58(0,24-1,41)$ & 10,7 & $1,94(1,21-3,11)$ \\
\hline Keine Defizite (Ref.) & & & 5,1 & 1,00 & 3,6 & 1,00 & 5,1 & 1,00 & 5,8 & 1,00 \\
\hline Skala $0-10$ & & & & $1,29(1,09-1,53)$ & & $1,46(1,19-1,79)$ & & $1,22(1,04-1,43)$ & & $1,45(1,25-1,68)$ \\
\hline
\end{tabular}

Signifikante Unterschiede sind durch Fettdruck hervorgehoben. 
Für alle Altersgruppen zeigt sich, dass Kinder aus der niedrigen Sozialstatusgruppe seltener gesund sind als Kinder aus der mittleren und hohen Statusgruppe. Auffällig ist allerdings, dass in der jüngsten Altersgruppe bei beiden Geschlechtern kein signifikanter Zusammenhang zwischen Sozialstatus und allgemeiner Gesundheit besteht. Bei männlichen Jugendlichen ergeben sich ebenfalls keine statistisch signifikanten Unterschiede nach Statusgruppenzugehörigkeit.

Mit Blick auf den Erwerbsstatus der Eltern fällt die Tendenz auf, dass Kinder und Jugendliche aus Familien, in denen mindestens ein Elternteil einer Vollzeitbeschäftigung nachgeht, häufiger eine gute Gesundheit besitzen als Kinder aus Familien, in denen dies nicht der Fall ist. Ein signifikanter Zusammenhang ist bei Jungen im Alter von 3 bis 13 Jahren und bei Mädchen in den Altersgruppen 7 bis 10 Jahre und 14 bis 17 Jahre festzustellen. Lediglich in der frühen Kindheit zeigen sich keine Unterschiede in der Gesundheit nach Erwerbsstatus.

Zwischen dem Übergewicht mindestens eines Elternteils und der elterlichen Einschätzung der Gesundheit besteht kein statistisch signifikanter Zusammenhang.

Wird in der Familienwohnung geraucht, stufen Eltern die Gesundheit ihres Kindes seltener als gut ein als Eltern, in deren Wohnung nicht geraucht wird. Ein signifikanter Zusammenhang lässt sich jedoch nur bei Jungen im Säuglings-, Kleinkind- und Vorschulalter feststellen.

Signifikante Einflüsse des mütterlichen Stillverhaltens zeigen sich bei Mädchen im Alter von 7 bis 10 Jahren und bei Jungen im Alter von 3 bis 10 Jahren. Bei den 7- bis 10-jährigen Jungen schneiden diejenigen, die zwar gestillt, aber nicht 6 Monate voll gestillt wurden, gesundheitlich am schlechtesten ab.

Eltern, die mit ihren Kindern in den ersten zwei Lebensjahren die U-Untersuchungen vollständig in Anspruch genommen haben, schätzen ihre Kinder eher als gesund ein als Eltern, deren Kinder nicht an allen Vorsorge-Untersuchungen teilgenommen haben. Signifikante Unterschiede zeigen sich bei den Jungen im Alter von 7 bis 13 Jahren und bei den Mädchen im Alter von 7 bis 17 Jahren.

Beim Familienklima gehen insbesondere starke Defizite mit einer nicht guten Gesundheit einher. Dies zeigt sich bei Mädchen und Jungen in allen Altersgruppen; lediglich im Grundschulalter bestehen bei den Jungen keine signifikanten Unterschiede.

Die Tab. 5 und 6 geben die Ergebnisse der multivariaten Analyse wieder. Die multivariate Analyse zeigt für die Altersspanne von 3 bis 17 Jahren die relativ hohe Bedeutung des Familienklimas für eine gute Gesundheit.

Eine weitere Auffälligkeit bei den Jungen stellt die Assoziation des beidseitigen Migrationshintergrunds mit einer nicht guten Gesundheit dar. Erst im Jugendalter verliert der Migrationshintergrund seinen Einfluss auf die allgemeine Gesundheit. Mit Blick auf die übrigen familialen Einflussfaktoren lassen sich bei den Jungen keine klaren Muster erkennen.

Bei den Mädchen besitzt im Säuglings- und Kleinkindalter keiner der untersuchten Faktoren Einfluss auf die elterliche Einschätzung der allgemeinen Gesundheit. Auch im Alter von 7 bis 10 Jahren besteht bei den Mädchen lediglich beim Familienklima ein signifikanter Zusammenhang. Außerdem zeigt das Gesundheitsverhalten der Eltern bei den Mädchen über alle Altersgruppen hinweg keinen von den anderen familialen Faktoren unabhängigen Effekt. Erwähnenswert ist zudem, dass der Sozialstatus in der gleichzeitigen Betrachtung aller Variablen nur noch bei den weiblichen Jugendlichen im Alter von 14 bis 17 Jahren ein signifikantes Odds Ratio erzielt. 
Wie die Pseudo- $\mathrm{R}^{2}$-Werte verdeutlichen, ist der Einfluss der untersuchten familialen Faktoren auf die durch die Eltern eingeschätzte allgemeine Gesundheit jedoch insgesamt recht gering.

Tab. 5: Multivariate Analyse der Bedeutung familialer Faktoren für eine nicht gute Gesundheit von Jungen, stratifiziert nach Altersgruppen (Odds Ratios und 95\%-Konfidenzintervalle

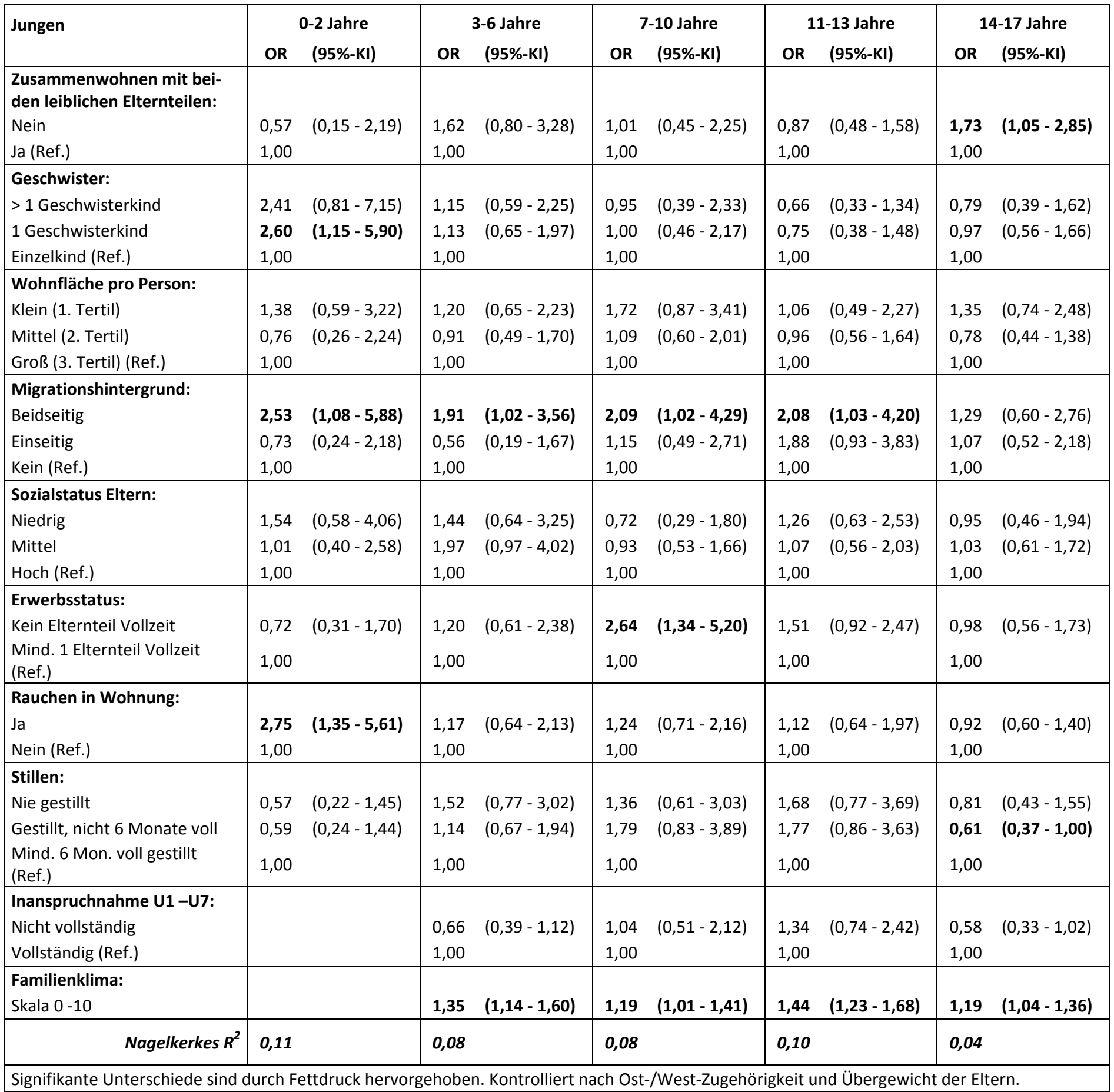


Tab. 6: Multivariate Analyse der Bedeutung familialer Faktoren für eine nicht gute Gesundheit von Mädchen, stratifiziert nach Altersgruppen (Odds Ratios und 95\%-Konfidenzintervalle)

\begin{tabular}{|c|c|c|c|c|c|c|c|c|c|c|}
\hline \multirow[t]{2}{*}{ Mädchen } & \multicolumn{2}{|r|}{ 0-2 Jahre } & \multicolumn{2}{|r|}{ 3-6 Jahre } & \multicolumn{2}{|c|}{ 7-10 Jahre } & \multicolumn{2}{|c|}{ 11-13 Jahre } & \multicolumn{2}{|c|}{ 14-17 Jahre } \\
\hline & OR & (95\%-KI) & OR & $(95 \%-K I)$ & OR & (95\%-KI) & OR & (95\%-KI) & OR & (95\%-KI) \\
\hline $\begin{array}{l}\text { Zusammenwohnen mit bei- } \\
\text { den leiblichen Elternteilen: } \\
\text { Nein } \\
\text { Ja (Ref.) }\end{array}$ & $\begin{array}{l}0,68 \\
1,00 \\
\end{array}$ & $(0,16-2,89)$ & $\begin{array}{l}0,59 \\
1,00 \\
\end{array}$ & $(0,24-1,48)$ & $\begin{array}{l}0,86 \\
1,00 \\
\end{array}$ & $(0,39-1,92)$ & $\begin{array}{l}2,03 \\
1,00 \\
\end{array}$ & $(1,05-3,94)$ & $\begin{array}{l}1,27 \\
1,00 \\
\end{array}$ & $(0,80-2,01)$ \\
\hline $\begin{array}{l}\text { Geschwister: } \\
>1 \text { Geschwisterkind } \\
1 \text { Geschwisterkind } \\
\text { Einzelkind (Ref.) }\end{array}$ & $\begin{array}{l}0,68 \\
1,17 \\
1,00 \\
\end{array}$ & $\begin{array}{l}(0,18-2,58) \\
(0,46-3,00)\end{array}$ & $\begin{array}{l}0,58 \\
0,52 \\
1,00 \\
\end{array}$ & $\begin{array}{l}(0,27-1,25) \\
(0,27-1,00)\end{array}$ & $\begin{array}{l}0,73 \\
0,93 \\
1,00 \\
\end{array}$ & $\begin{array}{l}(0,30-1,77) \\
(0,41-2,10)\end{array}$ & $\begin{array}{l}0,55 \\
1,21 \\
1,00 \\
\end{array}$ & $\begin{array}{l}(0,25-1,22) \\
(0,61-2,43)\end{array}$ & $\begin{array}{l}1,00 \\
1,16 \\
1,00 \\
\end{array}$ & $\begin{array}{l}(0,54-1,85) \\
(0,67-2,01)\end{array}$ \\
\hline $\begin{array}{l}\text { Wohnfläche pro Person: } \\
\text { Klein (1. Tertil) } \\
\text { Mittel (2. Tertil) } \\
\text { Groß (3. Tertil) (Ref.) }\end{array}$ & $\begin{array}{l}0,78 \\
1,07 \\
1,00 \\
\end{array}$ & $\begin{array}{l}(0,29-2,07) \\
(0,37-3,06)\end{array}$ & $\begin{array}{l}1,38 \\
1,00 \\
1,00 \\
\end{array}$ & $\begin{array}{l}(0,69-2,77) \\
(0,55-1,84)\end{array}$ & $\begin{array}{l}1,81 \\
0,64 \\
1,00 \\
\end{array}$ & $\begin{array}{l}(0,87-3,74) \\
(0,29-1,42)\end{array}$ & $\begin{array}{l}2,37 \\
1,03 \\
1,00 \\
\end{array}$ & $\begin{array}{l}(1,14-\mathbf{4 , 9 6}) \\
(0,54-1,99)\end{array}$ & $\begin{array}{l}1,61 \\
1,46 \\
1,00 \\
\end{array}$ & $\begin{array}{l}(0,98-2,65) \\
(0,91-2,36)\end{array}$ \\
\hline $\begin{array}{l}\text { Sozialstatus Eltern: } \\
\text { Niedrig } \\
\text { Mittel } \\
\text { Hoch (Ref.) } \\
\end{array}$ & $\begin{array}{l}1,41 \\
0,54 \\
1,00 \\
\end{array}$ & $\begin{array}{l}(0,49-4,02) \\
(0,20-1,48)\end{array}$ & $\begin{array}{l}1,59 \\
0,91 \\
1,00 \\
\end{array}$ & $\begin{array}{l}(0,74-3,38) \\
(0,48-1,71)\end{array}$ & $\begin{array}{l}2,11 \\
1,13 \\
1,00 \\
\end{array}$ & $\begin{array}{l}(0,85-5,22) \\
(0,50-2,55)\end{array}$ & $\begin{array}{l}0,87 \\
1,13 \\
1,00 \\
\end{array}$ & $\begin{array}{l}(0,35-2,20) \\
(0,54-2,38)\end{array}$ & $\begin{array}{l}1,46 \\
1,72 \\
1,00 \\
\end{array}$ & $\begin{array}{l}(0,74-2,88) \\
(1,01-2,93)\end{array}$ \\
\hline $\begin{array}{l}\text { Erwerbsstatus: } \\
\text { Kein Elternteil Vollzeit } \\
\text { Mind. } 1 \text { Elternteil Vollzeit } \\
\text { (Ref.) }\end{array}$ & $\begin{array}{l}1,24 \\
1,00\end{array}$ & $(0,37-4,15)$ & $\begin{array}{l}1,32 \\
1,00\end{array}$ & $(0,65-2,65)$ & $\begin{array}{l}1,17 \\
1,00\end{array}$ & $(0,52-2,65)$ & $\begin{array}{l}0,95 \\
1,00\end{array}$ & $(0,43-2,12)$ & $\begin{array}{l}1,67 \\
1,00\end{array}$ & $(1,07-2,60)$ \\
\hline $\begin{array}{l}\text { Rauchen in Wohnung: } \\
\text { Ja } \\
\text { Nein (Ref.) }\end{array}$ & $\begin{array}{l}0,77 \\
1,00 \\
\end{array}$ & $(0,30-1,99)$ & $\begin{array}{l}0,88 \\
1,00 \\
\end{array}$ & $(0,48-1,62)$ & $\begin{array}{l}0,78 \\
1,00 \\
\end{array}$ & $(0,44-1,38)$ & $\begin{array}{l}1,07 \\
1,00 \\
\end{array}$ & $(0,62-1,85)$ & $\begin{array}{l}0,82 \\
1,00 \\
\end{array}$ & $(0,53-1,28)$ \\
\hline $\begin{array}{l}\text { Familienklima: } \\
\text { Skala } 0-10\end{array}$ & & & 1,25 & $(1,03-1,52)$ & 1,34 & $(1,10-1,63)$ & 1,23 & $(1,04-1,45)$ & 1,36 & $(1,17-1,58)$ \\
\hline Nagelkerkes $R^{2}$ & 0,04 & & 0,07 & & 0,11 & & 0,07 & & 0,09 & \\
\hline
\end{tabular}

\section{Diskussion}

Der vorliegende Beitrag untersucht, in welchem Maße die Bedeutung familialer Faktoren für die allgemeine Gesundheit von Kindern und Jugendlichen nach Alter und Geschlecht variiert.

Mit Blick auf die in der Jugendgesundheitswissenschaft diskutierte These der „social equalisation in health“ (West 1997) ist festzuhalten, dass eine Abschwächung der Bedeutung familialer Faktoren im Jugendalter nicht generell festzustellen ist, sondern zwischen verschiedenen Faktoren differenziert werden muss. So kann insbesondere für das Familienklima 
für alle Altersgruppen (ausgenommen der 0- bis 2-Jährigen, für die keine Daten vorliegen) ein nahezu gleichbleibend hoher Zusammenhang gemessen werden.

Bei den Jungen lässt sich ferner eine hohe Bedeutung eines beidseitigen Migrationshintergrundes feststellen, der aber in der multivariaten Betrachtung im Jugendalter seine Signifikanz verliert. Dies könnte als Hinweis darauf gewertet werden, dass insbesondere in den früheren Lebensphasen sowohl kulturelle Aspekte als auch soziale Benachteiligungen, die eng mit dem Migrationsstatus verbunden sind, für die von den Eltern eingeschätzte Gesundheit von Bedeutung sind. Kritisch zu hinterfragen bleibt allerdings, ob Eltern mit Migrationserfahrung ggf. andere Maßstäbe zur Bewertung der Gesundheit ihres Kindes anlegen als Eltern ohne Migrationshintergrund.

In Hinblick auf den Sozialstatus fällt auf, dass dieser im Jugendalter nur bei den weiblichen Jugendlichen einen signifikanten und in der multivariaten Betrachtung vom Familienklima unabhängigen Einfluss besitzt. Für die weiblichen Jugendlichen lässt sich somit die These der Abschwächung der gesundheitlichen Ungleichheit nicht bestätigen. Auch bei Richter (2005) und Lampert (2010) zeigt sich ein Zusammenhang zwischen sozioökonomischen Faktoren und der allgemeinen Gesundheit in stärkerem Maße bei den weiblichen Jugendlichen. Richter und Hurrelmann (2008) führen dies auf eine höhere Sensibilität der Mädchen gegenüber sozialer Ungleichheit zurück. Klocke und Becker (2003) verweisen in diesem Zusammenhang auf das deutsche Schulsystem mit seinen drei Regelschulformen, dem gerade im Jugendalter eine eigenständige, ungleichheitsverstärkende Kraft zukommt. Letztlich bleibt allerdings ungeklärt, warum sich bei den männlichen Jugendlichen keine Unterschiede nach dem Sozialstatus der Eltern zeigen. Beachtenswert ist ferner, dass sich auch im Kleinkindalter kein signifikanter Einfluss des Sozialstatus ausmachen lässt. In Anlehnung an Walper (1999) kann vermutet werden, dass Säuglinge und Kleinkinder in stärkerem Maße vom Interaktionsklima der Familie als von der sozioökonomischen Lage beeinflusst werden. Hierzu erlaubt KiGGS allerdings keine Aussagen, da das Familienklima für dieses Alter nicht erhoben wurde.

Strukturellen Aspekten von Familie wie der Familienform und der Anzahl der Geschwister kommt mit Blick auf die elterliche Gesundheitseinschätzung nur eine geringe Bedeutung zu. Der Zusammenhang zwischen den verschiedenen Formen des familialen Zusammenlebens und der Gesundheit von Kindern und Jugendlichen ist eher indirekter Natur, da insbesondere materielle und auch zeitliche Ressourcen bei Alleinerziehenden in geringerem Maße vorhanden sind (Klocke \& Becker 2003; McMunn et al. 2001; Sweeting \& West 1995; Vingilis et al. 1998).

Auch der Erwerbsstatus der Eltern steht nicht in allen Altersgruppen in Zusammenhang mit der kindlichen Gesundheit. Hier kann in Anlehnung an Sleskova et al. (2006) vermutet werden, dass insbesondere eine lang andauernde Arbeitslosigkeit der Eltern negativ mit der subjektiven Gesundheit von Kindern und Jugendlichen assoziiert ist. Zur Länge der Arbeitslosigkeit enthält KiGGS jedoch keine Daten.

Mit Blick auf das Gesundheitsverhalten der Eltern zeigt sich - analog zur Literatur - kein konsistentes Bild. Es ist davon auszugehen, dass das Gesundheitsverhalten der Eltern durchaus einen, wenngleich auch eher geringen Einfluss auf den Gesundheitsstatus besitzt. Vermutlich ist es eher mit dem Gesundheitsverhalten der Heranwachsenden assoziiert und entfaltet darüber eine indirekte Wirkung auf die allgemeine Gesundheit (Case et al. 2002).

Insgesamt ist der Einfluss der untersuchten familialen Faktoren auf die durch die Eltern eingeschätzte allgemeine Gesundheit recht begrenzt. Die eingangs zitierten Forschungsbefunde legen nahe, dass weitere Faktoren der familialen Lebenswelt zu berücksichtigen sind, wie z.B. die Gesundheit der Eltern. Auch die Eltern-Kind-Beziehung, der Erziehungsstil, familiale Konflikte oder familiale Belastungen durch Suchtabhängigkeit eines 
Elternteils etc. könnten Einfluss auf die allgemeine Gesundheit haben. Eine wichtige moderierende Funktion kommt vermutlich auch der sozialen Einbindung der Familie in Kindertagesstätte, Schule, Nachbarschaft, Vereine etc. zu.

Da die psychische Gesundheit der Mutter einen starken Einfluss auf die elterliche Einschätzung der Gesundheit eines Kindes hat (s. Forschungsstand), kann nicht ausgeschlossen werden, dass die dargestellten Ergebnisse auf ein unterschiedliches Antwortverhalten der Eltern zurückzuführen sind. Die Überprüfung der Elternangaben anhand von ärztlichen Diagnosen durch Case und Paxson (2002) legt allerdings den Schluss nahe, dass die Elterneinschätzung als valide einzustufen ist.

Da in KiGGS neben der Elterneinschätzung der allgemeinen Gesundheit auch die Selbsteinschätzung der Jugendlichen erfasst wurde, können zu Zwecken der Validierung der Ergebnisse die Angaben verglichen werden. Der Vergleich ergibt, dass Eltern den Gesundheitszustand ihrer Kinder insgesamt positiver einschätzen als die 14- bis 17-Jährigen selbst. Dennoch zeigt sich bei den Jugendlichen - wie bei den Eltern - ein signifikanter Zusammenhang zwischen Familienklima und allgemeinem Gesundheitsstatus. Auch für den Sozialstatus sind in der bivariaten Betrachtung ähnliche Effektgrößen für die Eltern- und Selbsteinschätzung zu verzeichnen. In der multivariaten Analyse lässt sich jedoch auf der Basis der Selbsteinschätzungen sowohl bei den männlichen als auch bei den weiblichen Jugendlichen kein vom Familienklima unabhängiger Zusammenhang zwischen allgemeiner Gesundheit und Sozialstatus feststellen; während sich im Elternurteil bei den weiblichen Jugendlichen Unterschiede zeigen. Aufgrund der Unterschiede in der Beurteilung der allgemeinen Gesundheit durch Eltern und Heranwachsende werden der Verzicht auf die jugendlichen Selbstauskünfte und der ausschließliche Einbezug der Elterneinschätzungen in die vorgestellte Analyse rückblickend als sinnvoll eingestuft. Auch die im Forschungsstand berichteten divergierenden Ergebnisse können vermutlich in Teilen darauf zurückgeführt werden, dass einige Studien auf Eltern- und andere Studien auf Selbstangaben der Jugendlichen beruhen.

$\mathrm{Zu}$ beachten ist ferner, dass in die vorliegende Analyse lediglich Querschnittsdaten eingingen, die keine Aussagen zur Kausalität erlauben. Der Analyse liegt die Hypothese zugrunde, dass familiale Faktoren die Gesundheit von Kindern und Jugendlichen beeinflussen. Es ist aber auch möglich, dass gesundheitliche Einschränkungen von Heranwachsenden die familiale Lebensweise beeinflussen, so z.B. das Familienklima verschlechtern oder die Erwerbstätigkeit der Eltern einschränken.

Abschließend kann ebenfalls nicht geklärt werden, ob es sich bei den berichteten altersabhängigen Unterschieden tatsächlich um lebensphasenspezifische Besonderheiten oder eher um Kohortenunterschiede handelt. Aufgrund des Querschnittcharakters der Studie können nur Unterschiede zwischen verschiedenen Altersgruppen, nicht aber Veränderungen der Bedeutung von Familie für die Gesundheit eines Heranwachsenden zu verschiedenen Zeitpunkten beschrieben werden. Mit dem Ausbau der KiGGS-Studie zur Kohortenstudie wird dies zukünftig in Teilen möglich sein.

\section{Schlussfolgerungen}

Im Fokus der Gesundheitsförderung bei Kindern und Jugendlichen steht der Abbau sozial bedingter ungleicher Gesundheitschancen durch eine Verbesserung der sozialen Lebensbedingungen und eine größere gesellschaftliche Teilhabe sozial benachteiligter Kinder, Jugendlicher und Eltern (Bundesministerium für Familie, Senioren, Frauen und Jugend 2009; Bundesministerium für Gesundheit 2008, 2010). 
Auf der Basis der vorgestellten Ergebnisse kristallisiert sich als zentraler Ansatzpunkt einer familienorientierten Gesundheitsförderung die Verbesserung des innerfamilialen Klimas heraus. Gerade Strategien der Gesundheitsförderung, die die Verbesserung der sozialen Lebensbedingungen von benachteiligten Familien mit der Erweiterung von Verwirklichungschancen und Lebenskompetenzen von Eltern und Heranwachsenden und der Schaffung eines guten innerfamilialen Klimas verbinden, sind somit als zukunftsweisend einzustufen.

Während sich Kindertagesstätte und Schule bereits als wichtige gesundheitsfördernde Settings etablieren konnten, gibt es in der Praxis bisher nur wenige konkrete Ansätze für das "Setting Familie“. Dies wird u. a. auf die relativ hohe Abgeschlossenheit des Systems Familie zurückgeführt (Bals et al. 2008). Hier wäre eine bessere Verzahnung der Gesundheitsförderung mit der Kinder- und Jugendhilfe hilfreich, wie sie im 13. Kinder- und Jugendbericht gefordert wird (Bundesministerium für Familie, Senioren, Frauen und Jugend 2009), denn in der Kinder- und Jugendhilfe sind bereits Zugangswege zu sozial benachteiligten Familien entwickelt, die $u$. a. auf eine Verbesserung des Familienklimas zielen. Als positives Beispiel lassen sich die „Frühen Hilfen“ benennen, die sich durch eine enge Kooperation von Gesundheitssystem und Kinder- und Jugendhilfe auszeichnen und Eltern rund um die Geburt und in den ersten Lebensjahren des Kindes Unterstützung anbieten, die sowohl auf eine bessere soziale Teilhabe als auch ein gesundes familiales Miteinander ausgerichtet ist (Pott et al. 2010).

Darüber hinaus scheint eine stärkere Vernetzung der familialen Lebenswelt mit anderen pädagogischen Settings sinnvoll. Über eine stärkere Öffnung von Kindertagesstätte und Schule gegenüber Familie könnten zum einen Familien entlastende Angebote im Rahmen einer Ganztagsbetreuung unterbreitet werden, die so zu einer gewissen Verbesserung des Familienklimas beitragen können. Zum anderen könnten Bildungs- und Beratungsprozesse angeschoben werden, die die Erziehungs- und Lebenskompetenzen der Eltern und damit indirekt die Gesundheit der Kinder stärken. Eine „familienfokussierte Settingstrategie“ (Herlth 2008) erscheint somit insbesondere dann erfolgreich, wenn sie Familien in ihren Vernetzungen wahrnimmt und über diese den Familien „gesündere“ Handlungsoptionen anzubieten vermag, die Kindern und Jugendlichen eine gesunde physische, psychische und soziale Entwicklung ermöglichen.

\section{Literatur}

Almgren, G., Magarati, M., \& Mogford, L. (2009). Examining the influences of gender, race, ethnicity, and social capital on the subjective health of adolescents. Journal of Adolescence, 32(1), 109-133.

Bals, T., Hanses, A., \& Melzer, W. (2008). Gesundheitsförderung in pädagogischen Settings. Ein Überblick über Präventionsansätze in zielgruppenorientierten Lebenswelten. Weinheim: Juventa.

Bundesministerium für Familie, Senioren, Frauen und Jugend. (Hrsg.). (2005). 12. Kinder- und Jugendbericht. Bericht über die Lebenssituation junger Menschen und die Leistungen der Kinder- und Jugendhilfe in Deutschland. Berlin: BMFSFJ.

Bundesministerium für Familie, Senioren, Frauen und Jugend. (Hrsg.). (2009). 13. Kinder- und Jugendbericht. Bericht über die Lebenssituation junger Menschen und die Leistungen der Kinder- und Jugendhilfe in Deutschland. Mehr Chancen für gesundes Aufwachsen. Gesundheitsbezogene Prävention und Gesundheitsförderung in der Kinder- und Jugendhilfe. Berlin: BMFSFJ.

Bundesministerium für Gesundheit. (Hrsg.). (2008). Strategie der Bundesregierung zur Förderung der Kindergesundheit. Berlin: BMG. 
Bundesministerium für Gesundheit. (Hrsg.). (2010). Nationales Gesundheitsziel „Gesund aufwachsen: Lebenskompetenz, Bewegung, Ernährung". Berlin: BMG.

Burgess, S., Propper, C., \& Rigg, J. (2004). The impact of low income on child health: Evidence from a birth cohort study (LSE STICERD Research Paper No. CASE085). ttp://papers.ssrn.com/sol3/papers.cfm?abstract_id=1159316. Zugegriffen: 20. Mai 2010.

Call, K. T., \& Nonnemaker, J. (1999). Socioeconomic disparities in adolescent health: Contributing factors. Annals of the New York Academy of Sciences, 896, 352-355.

Case, A., \& Paxson, C. (2002). Parental behavior and child health. Health Affairs (Millwood), 21(2), 164-178.

Case, A., Lubotsky, D., \& Paxon, C. (2002). Economic status and health in childhood: The origins of the gradient. The American Economic Review, 92(5), 1308-1334.

Chen, E., Martin, A. D., \& Matthews, K. A. (2006). Socioeconomic status and health: Do gradients differ within childhood and adolescence? Social Science \& Medicine, 62(9), 2161-2170.

Chen, E., Matthews, K. A., \& Boyce, W. T. (2002). Socioeconomic differences in children's health: How and why do these relationships change with age? Psychological Bulletin, 128(2), 295-329.

Coneus, K., \& Spiess, K. (2008). The intergenerational transmission of health in early childhood (ZEW Discussion Paper No. 08-073). ftp://ftp.zew.de/pub/zewdocs/dp/dp08073.pdf. Zugegriffen: 20. Mai 2010.

Currie, A., Shields, M. A., \& Price, S. W. (2007). The child health/family income gradient: Evidence from England. Journal of Health Economics, 26(2), 213-232.

Dowd, J. B. (2007). Early childhood origins of the income/health gradient: The role of maternal health behaviors. Social Science \& Medicine, 65(6), 1202-1213.

Egle, U. T., Hardt, J., Nickel, R., Kappis, B., \& Hoffmann, S. O. (2002). Früher Streß und Langzeitfolgen für die Gesundheit. Wissenschaftlicher Erkenntnisstand und Forschungsdesiderate. Zeitschrift für Psychosomatische Medizin und Psychotherapie, 48, 411-434.

Emerson, E., Graham, H., \& Hatton, C. (2006). Household income and health status in children and adolescents in Britain. European Journal of Public Health, 16(4), 354-360.

Erhart, M., Holling, H., Bettge, S., Ravens-Sieberer, U., \& Schlack, R. (2007). Der Kinder- und Jugendgesundheitssurvey (KiGGS): Risiken und Ressourcen für die psychische Entwicklung von Kindern und Jugendlichen. Bundesgesundheitsbl Gesundheitsforsch Gesundheitsschutz, 50(5-6), 800-809.

Goodman, E. (1999). The role of socioeconomic status gradients in explaining differences in US adolescents' health. American Journal of Public Health, 89(10), 1522-1528.

Halldorsson, M., Kunst, A. E., Kohler, L., \& Mackenbach, J. P. (2000). Socioeconomic inequalities in the health of children and adolescents: A comparative study of the five Nordic countries. European Journal of Public Health, 10(4), 281-288.

Harris, M., Hollingsworth, B., Inder, B., \& Maitra, P. (2008). Re-examining the relationship between income and child health on both sides of the Atlantic. Technical report. (Research Paper 29). http://www.buseco.monash.edu.au/centres/che/pubs/rp29.pdf. Zugegriffen: 20. Mai 2010.

Herlth, A. (2008). Familiäre Einflussfaktoren auf die Kinder- und Jugendgesundheit und Konsequenzen für die Prävention. In T. Bals, A. Hanses, \& W. Melzer (Hrsg.), Gesundheitsförderung in pädagogischen Settings. Ein Überblick über Präventionsansätze in zielgruppenorientierten Lebenswelten (S. 29-50). Weinheim: Juventa. 
Hurrelmann, K. (2006). Einführung in die Sozialisationstheorie (9. Aufl.). Weinheim: Beltz.

Idler, E. L., \& Benyamini, Y. (1997). Self-rated health and mortality: A review of twenty-seven community studies. Journal of Health and Social Behavior, 38(1), 21-37.

Kamtsiuris, P., Lange, M., \& Schaffrath Rosario, A. (2007). Der Kinder- und Jugendgesundheitssurvey (KiGGS): Stichprobendesign, Response und Nonresponse-Analyse.

Bundesgesundheitsb/ Gesundheitsforsch Gesundheitsschutz, 50(5-6), 547-556.

Khanam, R., Nghiem, H. S., \& Connelly, L. B. (2009). Child health and the income gradient: Evidence from Australia. Journal of Health Economics, 28(4), 805-817.

Klocke, A., \& Becker, U. (2003). Die Lebenswelt Familie und ihre Auswirkungen auf die Gesundheit von Jugendlichen. In K. Hurrelmann, A. Klocke, W. Melzer, \& U. RavensSieberer (Hrsg.), Jugendgesundheitssurvey. Internationale Vergleichsstudie im Auftrag der Weltgesundheitsorganisation WHO (S. 183-241). Weinheim: Juventa.

Kolip, P. (1998). Familie und Gesundheit. In K. Hurrelmann \& U. Laaser (Hrsg.), Handbuch Gesundheitswissenschaften (S. 497-517). Weinheim: Juventa.

Kurth, B. M. (2007). Der Kinder- und Jugendgesundheitssurvey (KiGGS): Ein Überblick über Planung, Durchführung und Ergebnisse unter Berücksichtigung von Aspekten eines Qualitätsmanagements. Bundesgesundheitsbl Gesundheitsforsch Gesundheitsschutz, 50(5-6), 533-546.

Lampert, T. (2010). Gesundheitliche Ungleichheit: Welche Bedeutung kommt dem sozialen Status für die Gesundheit von Jugendlichen zu? In H. Hackauf \& H. Ohlbrecht (Hrsg.), Jugend und Gesundheit. Ein Forschungsüberblick (S. 44-65). Weinheim: Juventa

Lampert, T., Hagen, C., \& Heizmann, B. (2010). Gesundheitliche Ungleichheit bei Kindern und Jugendlichen in Deutschland. Berlin: Robert Koch-Institut.

Lange, M., Kamtsiuris, P., Lange, C., Schaffrath Rosario, A., Stolzenberg, H., \& Lampert, T. (2007). Messung soziodemographischer Merkmale im Kinder- und Jugendgesundheitssurvey (KiGGS) und ihre Bedeutung am Beispiel der Einschätzung des allgemeinen Gesundheitszustands. Bundesgesundheitsb/ Gesundheitsforsch Gesundheitsschutz, 50(5-6), 578-589.

McMunn, A. M., Nazroo, J. Y., Marmot, M. G., Boreham, R., \& Goodman, R. (2001). Children's emotional and behavioural well-being and the family environment: Findings from the health survey for England. Social Science \& Medicine, 53(4), 423-440.

Mullan, E., \& Currie, C. (2000). Socioeconomic inequalities in adolescent health. In C. Currie, K. Hurrelmann, W. Settertobulte, R. Smith, \& J. Todd (Hrsg.), Health and health behavior among young people. Health policy for children and adolescents no. 1 (S. 6572). Copenhagen: WHO Regional Office for Europe.

Murasko, J. E. (2008). An evaluation of the age-profile in the relationship between household income and the health of children in the United States. Journal of Health Economics, 27(6), 1489-1502.

Newacheck, P. W. (1989). Improving access to health services for adolescents from economically disadvantaged families. Pediatrics, 84(6), 1056-1063.

Piko, B., \& Fitzpatrick, K. M. (2001). Does class matter? SES and psychosocial health among Hungarian adolescents. Social Science \& Medicine, 53(6), 817-830.

Pott, E., Fillinger, U., \& Paul, M. (2010). Herausforderungen bei der Gesundheitsförderung im frühen Kindesalter. Bundesgesundheitsbl Gesundheitsforsch Gesundheitsschutz, 53(11), 1166-1172.

Rahkonen, O., Arber, S., \& Lahelma, E. (1995). Health inequalities in early adulthood: A comparison of young men and women in Britain and Finland. Social Science \& Medicine, 41(2), 163-171. 
Richter, M. (2005). Bedeutung sozialer Ungleichheit für die Gesundheit im Jugendalter. Gesundheitswesen, 67(10), 709-718.

Richter, M., \& Hurrelmann, K. (2008). Gesundheitliche Ungleichheit bei Kindern und Jugendlichen: Aktuelle Befunde und Erklärungsansätze. Public Health Forum, 16(59), 21.e2121.e22.

Schenk, L., Neuhauser, H., Ellert, U., Poethko-Müller, C., Kleiser, C., \& Mensink, G. (2008). Kinder- und Jugendgesundheitssurvey (KiGGS 2003-2006): Kinder und Jugendliche mit Migrationshintergrund in Deutschland. Berlin: Robert Koch-Institut.

Schneewind, K., Beckmann, M., \& Hecht-Jackl, A. (1985). Familienklima-Skalen. München: Institut für Psychologie - Persönlichkeitspsychologie und Psychodiagnostik. Ludwig Maximilians Universität.

Siahpush, M., \& Singh, G. K. (2000). A multivariate analysis of the association between social class of origin and current social class with self-rated general health and psychological health among 16-year-old Australians. Australian and New Zealand Journal of Medicine, 30(6), 653-659.

Sleskova, M., Salonna, F., Geckova, A. M., Nagyova, I., Stewart, R. E., van Dijk, J. P., et al. (2006). Does parental unemployment affect adolescents' health? The Journal of Adolescent Health, 38(5), 527-535.

Spencer, N. J. (2006). Social equalization in youth: Evidence from a cross-sectional British survey. European Journal of Public Health, 16(4), 368-375.

Sweeting, H., \& West, P. (1995). Family life and health in adolescence: A role for culture in the health inequalities debate? Social Science \& Medicine, 40(2), 163-175.

Torsheim, T., Currie, C., Boyce, W., Kalnins, I., Overpeck, M., \& Haugland, S. (2004). Material deprivation and self-rated health: A multilevel study of adolescents from 22 European and North American countries. Social Science \& Medicine, 59(1), 1-12.

van der Lucht, F., Groothoff, J. W., Koopmans, P. C., \& van der Heuvel, W. J. A. (1992). Socioeconomic health differences among children aged 10-11 in the Netherlands. European Journal of Public Health, 2(1), 24-28.

Vingilis, E., Wade, T. J., \& Adlaf, E. (1998). What factors predict student self-rated physical health? Journal of Adolescence, 21(1), 83-97.

Vuille, J. C., \& Schenkel, M. (2001). Social equalization in the health of youth. The role of the school. European Journal of Public Health, 11(3), 287-293.

Walper, S. (1999). Auswirkungen von Armut auf die Entwicklung von Kindern. In A. Lepenies, G. Nunner-Winkler, G. E. Schäfer, \& S. Walper (Hrsg.), Kindliche Entwicklungspotentiale. Normalität, Abweichung und ihre Ursachen. Materialien zum 10. Kinder- und Jugendbericht, Bd. 1 (S. 291-350). München: Deutsches Jugendinstitut.

Watanabe, M., Nakamura, K., Fukuda, Y., \& Takano, T. (2006). Association of parental and children behaviors with the health status of preschool children. Preventive Medicine, 42(4), 297-300.

West, P. (1988). Inequalities? Social class differentials in health in British youth. Social Science \& Medicine, 27(4), 291-296.

West, P. (1997). Health inequalities in the early years: Is there equalisation in youth? Social Science \& Medicine, 44(6), 833-858.

West, P., Macintyre, S., Annandale, E., \& Hunt, K. (1990). Social class and health in youth: Findings from the west of Scotland twenty-07 study. Social Science \& Medicine, 30(6), 665-673. 